Radio Interferometry: Theory, Techniques and Applications, LAU Coll. 131, ASP Conference Series, Vol. 19, 1991.

T.J. Comwell and R.A. Perley (eds.)

THE LINK TO EXTRAGALACTIC SOURCES : OBSERVATIONS OF RADIO STARS

ALAIN BAUDRY and YVES REQUIEME

Observatoire de l'Université de Bordeaux, B.P. 89, 33270 Floirac, France

ABSTRACT Optical positions obtained with the Bordeaux automatic meridian circle (accuracy $\sim 0.05$ ") are compared with VLA positions taken from the literature. Alignment of the FK5 and VLA frames are discussed, and other projects (HIPPARCOS, VLBI) are briefly presented.

\title{
INTRODUCTION
}

The best materialization of a quasi-inertial reference system rests on VLBI observations of extragalactic sources with milliarcsecond (mas) accuracy. However this system is not dense (less than 300 radio sources) and has faint or no optical counterparts. In the optical domain the astrometric space mission HIPPARCOS will provide positions for about 100000 stars with $\sim 2$ mas accuracy. This dense and rigid system might be rotating with respect to the extragalactic system. Therefore the unification of the radio and reference systems appears as an important task of modern astrometry.

Radio stars, optically bright to be accessible to HIPPARCOS (mV $\leq 11$ ), provide a direct way to link the radio and optical systems. Obviously the quality of the link depends on the coincidence of the radio and optical centers.

\section{RADIO STARS}

We consider here stars exhibiting continuum radio emission and stars with strong circumstellar maser emission. The first group of stars is not homogeneous and contains quasi-thermal emission shell objects as well as highly non-thermal emitters with radio flares. The VLA was used to determine the radio positions of 20 stars (Florkowski et al., 1985) with respect to the VLA extragalactic reference frame; the internal precision is about 0.03 ". In VLBI, only the core emitting region can be detected thus restricting the sample of stars to the highly non-thermal emitters (RS CVn stars). Positions of 8 stars with respect to the VLBI extragalactic frame are given by Lestrade et al. (1988) ; the accuracy varies from 0.07 " to 0.002 ". The second group of stars is composed of late $\mathrm{M}$ Mira-type variables and of $\mathrm{M}$ supergiants with $\mathrm{OH}, \mathrm{H}_{2} \mathrm{O}$ or $\mathrm{SiO}$ maser emission in expanding envelopes. Because the maser spots detected in VLBI are extremely compact they are of astrometric interest, especially $\mathrm{SiO}$ which is excited close to the stellar photosphere (e.g. Baudry et al., 1990). Accurate positions ( 0.10"- 0.15") of masers were obtained at the VLA (e.g. Bowers et al., 1989) and in SiO (Wright et al., 1990). 


\section{COMPARISON OF OPTICAL AND RADIO POSITIONS}

The Bordeaux automatic meridian circle was used between 1984 and 1990 to observe about 70 bright radio continuum and maser stars relative to the FK5 stars. The standard deviation of an observation is $0.11^{\prime \prime}$ and $0.16^{\prime \prime}$ in right ascension and declination respectively. (The deviation increases to $0.25^{\prime \prime}$ at $\delta=-25^{\circ}$.) In general each radio star was observed more than 30 times so that a conservative estimate of the position accuracy for stars in the Northern hemisphere is $\pm 0.05^{\prime \prime}$. The stellar parallax if known was removed from each observation. Systematic errors reaching $0.1^{\prime \prime}$ near $\delta=+60^{\circ}$ are present in the FK5, but no corrections were made to bring our observations closer to the FK5.

Our optical positions were first published by Baudry et al. (1990) and Morrison et al. (1990). We add here VX Sgr, a strongly masing supergiant, HR1099, $\alpha$ Sco A, 9 Sgr, HD50896, P Cyg, Cyg OB2 No 8A, 9 and 12, and $\sigma$ CrB A (Requième et al., in preparation).

\section{Continuum Stars}

Most radio positions are taken from Table 2 of Florkowski et al. ; for other references see our Table 1. We transformed the radio positions from B1950.0 to J2000.0 following the procedure of Aoki et al. (1983); therefore the J2000.0 VLA coordinates are directly comparable to the FK5 catalog. Note that the VLA calibrator epoch is 1979.9 where Perley (1982) observed the primary VLA calibrators.

The optical minus radio differences are given in Table 1 . They do not show any systematic effects with the coordinates. An unexplained right ascension offset is present in $54 \mathrm{Cam}$. Excluding $54 \mathrm{Cam}$, the means of the differences are $\Delta \alpha \cos \delta=-0.004 "$ and $\Delta \delta=+0.016^{\prime \prime}$ with mean square errors $\pm 0.08^{\prime \prime}$ and $\pm 0.09^{\prime \prime}$ respectively. The optical accuracy is $\pm 0.05^{\prime \prime}$ and the internal precision of the VLA positions is $\sim 0.03^{\prime \prime}$ in general. However we note that discrepancies $~ 0.06 "-0.08$ " exist between the positions of the two VLA calibrators (B1950) used for 9 Sgr and HR1099, and the positions given in the last version of the VLA calibration catalog. $\alpha$ Sco $\mathrm{A}$ is another interesting case. The declination discrepancy for the associated calibrator (1622-297) amounts to 0.24 " which is well compensated by our -0.23 " in Table 1 ; therefore the optical and radio objects coincide.

Various physical effects could explain some individual optical-radio differences : unknown or poor proper motions (e.g. VX Sgr or FR Sct); extended radio emission (due for example to the proximity of an early star as in $\alpha$ Sco); visual double systems (UX Ari, Cyg OB2 No 5) or interacting stars, etc. It is clear that speckle-interferometry programs would help selecting the best astrometric optical candidates. In this work we have included, $\alpha$ Sco A, $\sigma$ CrB A and HR1099 although they have nearby companions. For the rotating knife-edge detector of our meridian circle (field of view $\sim 13^{\prime \prime}$ ) the calculated corrections to the positions depend on the magnitude difference of the components, the position angle and the diameter of the star images (all parameters which are usually not well known).

\section{Maser Stars}

Our optical positions of maser stars were compared to the most accurate radio positions taken from the published literature. All radio positions refer to extragalactic sources pertaining to the VLA catalog although the calibrator positions are not always given. Excluding VY CMa (see below), we derive the means $\Delta \alpha \cos \delta=$ $+0.11^{\prime \prime}$ and $\Delta \delta=-0.02^{\prime \prime}$ with mean square errors of $\pm 0.16^{\prime \prime}$ and $0.14 "$ respectively. 
TABLE 1 Differences optical (Bordeaux meridian circle) minus radio (VLA) positions of radio continuum stars at the cpoch of the radio observations*

\begin{tabular}{|c|c|c|c|c|c|c|c|}
\hline \multirow[t]{2}{*}{ Star } & \multirow[t]{2}{*}{$\begin{array}{c}\text { DEC } \\
(2000.0)\end{array}$} & \multicolumn{2}{|c|}{ Bordcaux ${ }^{a}-V L A^{b}$} & \multirow[t]{2}{*}{ Star } & \multirow[t]{2}{*}{$\begin{array}{c}\text { DEC } \\
(2000.0)\end{array}$} & \multicolumn{2}{|c|}{ Bordcau $^{a}-$ VLA $^{b}$} \\
\hline & & $\Delta \alpha \cos \delta$ & $\Delta \delta$ & & & $\Delta \alpha \cos \delta$ & $\Delta \delta$ \\
\hline UX Ari & $+28^{\circ} 42^{\prime}$ & -0.12 & $+0: 05$ & ER Sct & $-12^{\circ} 40^{\circ}$ & +0.05 & -0.05 \\
\hline HR 1099 & +035 & -0.05 & -0.02 & $\beta$ Lyr & +3321 & +0.02 & +0.05 \\
\hline b Per & $+50 \quad 17$ & -0.12 & -0.05 & P Cyg & +3801 & -0.18 & +0.08 \\
\hline HD 50896 & -2355 & -0.08 & +0.08 & $B D+433571$ & +4351 & +0.06 & +0.14 \\
\hline KO Pup & -1431 & -0.06 & -0.22 & $C_{Y g}$ OB2 $N^{\circ} 5$ & $+41 \quad 18$ & +0.07 & +0.08 \\
\hline 54 Cam & +5716 & +0.52 & 0.00 & $C_{Y g}$ OB2 $N^{\circ} 12$ & $+41 \quad 14$ & +0.15 & +0.05 \\
\hline RS CVn & +3556 & -0.03 & +0.14 & CYg OB2 $N^{\circ} 9$ & +4115 & -0.06 & +0.04 \\
\hline EK Com & +2413 & +0.07 & +0.03 & CYg OB2 $N^{\circ} 8 \mathrm{~A}$ & +4118 & -0.09 & +0.09 \\
\hline HR 5110 & +3710 & -0.01 & +0.03 & vV Cep & +6337 & +0.02 & +0.03 \\
\hline$\sigma$ CrB A & +3351 & -0.04 & -0.02 & RT LaC & +4353 & +0.02 & -0.03 \\
\hline$\alpha \operatorname{Sco} A$ & -2625 & +0.04 & -0.23 & AR Lac & +4544 & +0.01 & +0.02 \\
\hline$z$ Her & +1508 & +0.02 & +0.03 & SZ PSC & +240 & +0.17 & -0.03 \\
\hline $9 \mathrm{Sgr}$ & $-24 \quad 21$ & +0.03 & +0.06 & HD 224085 & $+28 \quad 38$ & $+0 \quad 02$ & +0.06 \\
\hline
\end{tabular}

* Optical positions from Morrison ct al. (1990) and from this work. Radio positions from Florkowski et al. (1985), Johnston ct al. (1985), de Vegt (1982) and Abbott et al. (1981)

a) $\mathrm{j} 2000.0$ optical positions

b) B1950.0 radio positions transformed to, 2000.0

TABLE 2 Comparison of optical (Bordcaux mcridian circlc) positions with radio positions of mascr stars*

\begin{tabular}{|c|c|c|c|c|c|c|c|c|c|}
\hline \multirow[t]{2}{*}{ Star } & \multicolumn{6}{|c|}{ Radio positions ${ }^{a}$} & \multirow[t]{2}{*}{ Moleculc } & \multicolumn{2}{|c|}{ Optical - Radio } \\
\hline & \multicolumn{3}{|c|}{ RA(1950) } & \multicolumn{3}{|c|}{$\operatorname{DEC}(1950)$} & & $\Delta \alpha \cos \delta$ & $\Delta \delta$ \\
\hline - Cet & \multicolumn{3}{|c|}{$2^{h} 16^{m} 49^{s} .076$} & \multicolumn{3}{|c|}{$-3^{\circ} 12 \cdot 22: 60$} & Sio & $0: 06$ & 0.00 \\
\hline S Per & 2 & 19 & 15.090 & 58 & 21 & 33.40 & $1667 \mathrm{OH}$ & 0.02 & 0.00 \\
\hline U ori & 5 & 52 & 50.909 & 20 & 10 & 05.85 & $1612 \mathrm{OH}$ & -0.06 & 0.03 \\
\hline \multirow[t]{3}{*}{ VY CMa } & 7 & 20 & 54.739 & -25 & 40 & 12.27 & Sio & -0.92 & -0.62 \\
\hline & & & 54.74 & & & 12.4 & $1612 \mathrm{OH}$ & -0.93 & -0.40 \\
\hline & & & 54.66 & & & 12.2 & $\mathrm{H}_{2} \mathrm{O}$ & -0.19 & -0.66 \\
\hline$R$ Leo & 9 & 44 & 52.210 & 11 & 39 & 40.26 & sio & 0.18 & -0.16 \\
\hline w Hya & 13 & 46 & 12.033 & -28 & 07 & 09.09 & sio & 0.08 & 0.25 \\
\hline $\mathrm{RX} \mathrm{BOO}$ & 14 & 21 & 56.718 & 25 & 55 & 47.17 & $\mathrm{H}_{2} \mathrm{O}$ & -0.01 & 0.02 \\
\hline$S \mathrm{CrB}$ & 15 & 19 & 21.54 & 31 & 32 & 45.7 & $1665 / 67 \mathrm{OH}$ & 0.41 & 0.16 \\
\hline U Her & 16 & 23 & 34.692 & 19 & 00 & 17.73 & $1667 \mathrm{OH}$ & 0.00 & 0.02 \\
\hline$v x \operatorname{sg} x$ & 18 & 05 & 02.959 & -22 & 13 & 55.58 & sio & 0.25 & 0.27 \\
\hline R AqI & 19 & 03 & 57.687 & 8 & 09 & 07.87 & $\mathrm{H}_{2} \mathrm{O}$ & 0.12 & 0.07 \\
\hline$x$ cyg & 19 & 48 & 38.470 & 32 & 47 & 10.06 & sio & -0.15 & -0.07 \\
\hline RR Aql & 19 & 55 & 00.309 & -2 & 01 & 17.48 & $1612 \mathrm{OH}$ & 0.03 & -0.24 \\
\hline $\mathrm{R} A \mathrm{Ag}$ & 23 & 41 & 14.252 & -15 & 33 & 43.26 & sio & 0.25 & 0.00 \\
\hline \multirow[t]{2}{*}{$\mathrm{R}$ Cas } & 23 & 55 & 52.000 & 51 & 06 & 37.76 & Sio & 0.37 & -0.01 \\
\hline & & & 51.91 & & & 38.2 & $1665 / 67 \mathrm{OH}$ & 0.36 & -0.72 \\
\hline
\end{tabular}

- Radio positions are transformed to 2000.0 and compared with optical positions at the epoch of the radio obscrvations

a) For references sce Table 5 of Baudry et al. (1990), and Wright et al. (1990) 
The scatter in the optical-radio differences is larger than for the data in Table 1, a result which can partly be attributed to less accuracy in the radio positions of masers.

Extended and complex circumstellar maser envelopes also contribute to the optical-radio differences. This is the case for the SiO maser in VY CMa where large position offsets are observed. Furthermore this star is embedded in a small optical nebulosity containing several condensations; it is not suitable for astrometry. In addition, we cannot exclude that some optical positions of late type stars are perturbed by the presence of a nearby companion (e.g. o Cet a binary with components separated by $\sim 0.8 "$ ).

\section{FUTURE WORKS}

The extragalactic sources used as references for the star positions discussed here pertain to the catalog of 234 compact radio/optical objects (Argue et al., 1984) with accuracy $\leq 0.01$ ". On the other hand the present accuracy of the FK5 catalog is only $0.04 "-0.05 "$ ". Therefore aligning the FK5 and VLA frames with accuracy $\sim 0.05^{\prime \prime}$ should be feasible. We have shown however that this requires a careful analysis of the optical and radio structures of stars. Then, provided that enough stars are observed, the remaining optical-radio differences related to structural effects should cancel out in the statistical sense. In this context, it is interesting to note that several tens of maser stars are strong enough to be observed with radio interferometers and that the associated stars are accessible to the HIPPARCOS satellite. One must preferably select late type stars with low mass loss rates, i.e. with compact envelopes.

Tying together the optical and radio reference frames with 0.05 " accuracy must be regarded as an intermediate step allowing to test the suitability and, indirectly, the nature of the link stars, whereas the mas level is required at a further stage to align the HIPPARCOS and VLBI frames. We have selected $50 \mathrm{SiO}$ maser stars well distributed over the celestial sphere for observations with HIPPARCOS. Recently, mas spot sizes were discovered with European VLBI baselines up to $1350 \mathrm{~km}$ in several $\mathrm{SiO}$ stars (Colomer et al., this colloquium), and positions of $\mathrm{SiO}$ masers relative to nearby quasars are planned.

\section{REFERENCES}

Abbott, D.C., Bieging, J.H., Churchwell, Ed. 1981, Ap. J., 250, 645

Aoki, S., Sôma, S., Kinoshita, H., Inoue, K. 1983, A. \& A., 128, 263

Argue, A.N. et al. 1984, A. \& A., 130, 191

Baudry, A., Mazurier, J.M., Périé, J.P., Requième, Y., Rousseau, J.M. 1990, A. E A., 232,258

Bowers, P.F., Johnston, K.J., de Vegt, C. 1989, Ap. J., 340, 479

De Vegt, C. 1982, A. \& A., 109, 282

Florkowski, D.R., Johnston, K.J., Wade, C.M., de Vegt, C. 1985, A. J., 90, 2381

Johnston, K.J., Wade, C.M., Florkowski, D.R., de Vegt, C. 1985, A. J., 90, 1343

Lestrade, J.F., Niell, A.E., Preston, R.A., Mutel, R.L. 1988, A. J., 96, 1746

Morrison, L.V., Argyle, R.W., Requième, Y., Mazurier, J.M. 1990, A. \& A., 236, 256

Perley, R.A. 1982, A. J., 87, 859

Wright, M.C.H., Carlstrom, J.E., Plambeck, R.L., Welch, W.J. 1990, A. J., 99, 1299 